\title{
ToA-TS: Time of Arrival based Joint Time Synchronization and Tracking for Mobile Underwater Systems
}

\author{
Jinwang $\mathrm{Yi}^{\mathrm{a}}$, Diba Mirza ${ }^{\mathrm{b}}$, Ryan Kastner $^{\mathrm{b}}$, Curt Schurgers $^{\mathrm{b}}$, Paul Roberts ${ }^{\mathrm{c}}$, \\ Jules Jaffe ${ }^{\mathrm{C}}$ \\ ${ }^{a}$ Xiamen University, China \\ ${ }^{b}$ University of California, San Diego \\ ${ }^{c}$ Scripps Institution for Oceanography
}

\begin{abstract}
Time synchronization and localization are key requirements for distributed underwater systems consisting of numerous low-cost submersibles. In these systems, submersibles are highly resource constrained and typically have limited acoustic communication capability. We investigate the problem of tracking submersibles that only have the capability of receiving acoustic signals. Traditional Long Base Line (LBL) systems track the location of submersibles by providing a GPS-like infrastructure that consists of a few reference beacons at known locations. In these systems the unknown positions of submersibles are estimated from beacon transmissions using time-difference-of-arrival (TDoA) based localization. As such TDoA makes the key assumption that beacon transmissions occur nearly concurrently in time. While this assumption is ensured in small LBL deployments it does not hold as the size of the system scales up. In this paper we identify scenarios where signals from multiple beacons are significantly lagged in time. We further identify the motion of the submersible between signal arrivals as a key factor that deteriorates the performance of TDoA, when transmissions are not concurrent. To address this problem we propose to track the submersible while performing time-synchronization. Our proposed technique,
\end{abstract}

Email addresses: j1yi@eng.ucsd.edu (Jinwang Yi), dimirza@eng.ucsd.edu (Diba Mirza), kastner@ucsd.edu (Ryan Kastner), cschurgers@ucsd.edu (Curt Schurgers), paulr@mpl.ucsd.edu (Paul Roberts), jules@mpl.ucsd.edu (Jules Jaffe)

Preprint submitted to Journal of Ad Hoc Networks

October 5, 2014

(C) 2014. This manuscript version is made available under the Elsevier user license http://www.elsevier.com/open-access/userlicense/1.0/ 
called Time of Arrival based Tracked Synchronization (ToA-TS) essentially extends GPS like localization for scenarios where beacon transmissions are not concurrent and submersibles are not capable of two-way communication. We show the benefit of our proposed scheme by comparing its performance to other localization techniques using experimentally obtained data.

Keywords: Underwater Networks, Real-time Tracking, Acoustic Networks

\section{Introduction}

Mobile underwater submersibles are widely used in many oceanographic applications. As submersibles become more agile, they present new opportunities to study oceanic phenomena that are dynamic and vary at smaller spatial scales

5 (tens to hundreds of meters). To aid such exploration there has been a growing interest to deploy numerous low-cost and compact submersibles as swarms that operate in a distributed and coordinated fashion. A crucial feature of such distributed systems is that the cost and capabilities of individual submersibles are scaled down in order to scale up the spatial extent and density of the sampling system. As a result, submersibles are highly energy constrained, and typically have very short-range acoustic communication or in many cases only have the ability to act as passive receivers.

Determining the location and time of submersibles underwater in a global frame of reference are two key requirements of distributed systems. Global position and time can be easily obtained from GPS for terrestrial systems. However, due to the unavailability of GPS underwater, traditional underwater acoustic navigation techniques typically rely on a GPS-like infrastructure to track submersibles. This infrastructure consists of a few reference beacons (usually four) that are deployed in precise configurations as in Long Baseline Systems ${ }_{20}$ (LBL) [1]. The beacons essentially act as GPS satellites for underwater by deducing their own position and time from GPS. The submersibles position and local time are jointly estimated from measurements of the time-difference-of- 
arrival (TDoA) of acoustic signals that are transmitted by the beacons ${ }^{1}$. TDoA is especially relevant to tracking and synchronizing low-cost submersibles that have limited acoustic communication capability and can seldom transmit to surface beacons. However, TDoA makes two crucial assumptions during the interval that the time-of-arrival measurements are obtained (a) the submersible is stationary, and (b) the difference between the global time according to beacons and the local time of the submersible, (i.e., clock offset) remains constant. clock offset changes at an unknown rate, which is known as the clock drift. Therefore, while the assumptions made by TDoA do not hold in general, they are satisfied in practical LBL systems by ensuring that the submersible receives four signals in succession over a short time window as depicted in Figure 1(a). 35 In such a scenario, the movement of the submersible is negligible during the signaling period. Similarly, its clock offset is virtually constant (i.e., the effect of drift is negligible). The problem that we address in this paper is time synchronization and tracking submersibles when signals from multiple beacons no longer arrive concurrently, but rather arrive over a much longer time epoch, $T$ 40 as depicted in Figure 1(b). We will explain shortly why this problem arises in the context of swarm systems. The key point is that when signals no longer arrive concurrently at each submersible, two effects come into play (that are ignored by TDoA based localization): the submersible can significantly move between signal arrivals and the clock offset may not remain constant over the 45 signaling period due to the cumulative effect of clock drift. In this paper we identify scenarios where the error introduced due to these two factors becomes significant for a nominal range of relevant parameters (a detailed discussion is presented in Section 3). When there is significant motion between signal ar-

\footnotetext{
${ }^{1}$ Other acoustic navigation techniques are also possible within this basic framework. We discuss these techniques in related work (Section 7). However, these approaches typically rely on bi-directional communication with beacons. Therefore, they are not suitable for tracking submersibles with only receive capability.
} 
rivals, in order to time synchronize the submersible, it has to be additionally tracked over the time epoch T. Therefore, the key problem that we will solve is joint time synchronization and tracking of submersibles based only on oneway acoustic transmissions from beacons. In the next section we delineate why this problem arises in the context of swarm systems and discuss our solution strategy. Our proposed technique, called Time of Arrival based Tracked Synchronization (ToA-TS) extends GPS like localization for scenarios where beacon transmissions are not concurrent and submersibles are not capable of two-way communication.

In this paper we extend on our previous work in [2], where we proposed a joint approach to time synchronization and localization using the framework of 60 factor-graphs. In particular, we improve the run-time of our original factorgraph based algorithm by bootstrapping from an analytically obtained initial solution. In addition we present an extensive comparison of the performance of ToA-TS to other tracking techniques using experimentally obtained data.

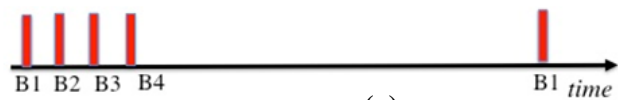

(a)

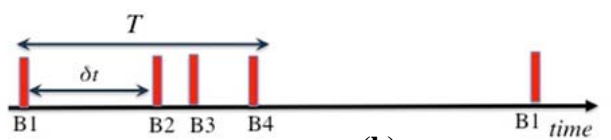

(b)

Figure 1: Signals received at a submersible from four beacons in (a) LBL systems and (b) Swarm systems

\section{Problem Motivation}

In scenarios where beacon transmissions are spread over a time window $T$, two key effects come into play. However, prior to discussing these effects in more detail, in this section we discuss why signals from transmissions are likely to occur over longer time epochs in swarm systems compared to traditional LBL deployments. 
Traditional LBL systems are designed for small deployments consisting of a few beacons (typically four), whose acoustic transmissions can be received by all submersibles within the deployment. As such LBL provides sufficient localization accuracy for such deployments. This is because signals from few beacons can be scheduled to occur within a short time window. Since all the 75 submersibles are within the communication range of the beacons, these signals also arrive at near concurrent times at each submersible as shown in Figure 1(a). However, as the spatial extent and size of the system is scaled up, this scenario changes.

As the spatial extent of the deployment increases, more beacons are required to provide sufficient acoustic coverage for localizing submersibles. Further, these beacons may not be deployed in precise configurations. This makes it challenging to avoid collisions while meeting the signal concurrency requirement of traditional LBL systems. One possible solution to this problem involves using orthogonal signals for the beacon transmissions. However, even with this approach, as the system scales, the number of beacons would exceed the number of orthogonal signal dimensions. Further, such a solution is not possible in many narrow-band systems due to hardware constraints. We consider situations where orthogonal signaling is not employed, either by design or due to scalability. In such systems, transmissions from multiple beacons have to be suf90 ficiently lagged over a time epoch, $T$ to avoid collisions. Given that propagation delays are in the order of seconds, the time lag between beacon transmissions is likely to be in the order of tens of seconds or more. As a result transmissions from multiple beacons can no longer be scheduled to occur concurrently. The crucial point here is that while we still propose a system where all beacons try to schedule their transmission as close as possible in time, these transmissions cannot be concurrent due to the size of the network. A submersible within this network can only receive signals from a subset of beacons. Further, this subset changes due to the motion of the submersible and possibly that of the beacons [3]. Therefore, the time lag, $\delta t$ between the signals received by a submersible from a random subset of beacons can be significant as depicted in Figure 1(b). 
In such scenarios two additional factors come into play, which were previously ignored by TDoA, namely, the motion of the submersible and the drift in its local clock between signal arrivals. These uncertainties are different from random measurement errors. We will make this distinction as part of the problem formulation in section 4 . Later in the same section we discuss scenarios where the two factors under consideration, namely the motion of the submersible and the drift in the local clock can significantly affect the performance of TDoA localization, which assumes concurrent transmissions.

\section{TDoA Localization with Non-concurrent Signal Arrivals}

First, we briefly describe the time-difference-of-arrival (TDoA) method, which is typically used in LBL systems. In TDoA based localization the difference in the measured time of arrivals of signals received from a pair of beacons translates to the difference in range estimates with those beacons. As a result each estimate of time-difference gives rise to a hyperbola for the unknown position of the submersible. A unique estimate of the submersibles position can be obtained by intersecting three such hyperbolas (obtained from four beacon transmissions). However, this technique requires that beacons transmit at near concurrent times. For the scenario where transmissions are lagged, this approach essentially neglects the motion and clock drift of the submersible between signal arrivals. In reality, both these factors add an uncertainty to the measured time differences. As a result, each hyperbola thus obtained does not represent the submersibles position at any particular time. Therefore, the hyperbolas no longer intersect at a common point. A least squares approach can still be used to obtain an estimate for the position. However, these uncertainties are likely to degrade the performance of TDoA based localization. Furthermore, as we will show in the next section, the uncertainty due to the unknown clock offset and that due to vehicle motion can be estimated with a more precise formulation of the problem and velocity measurements obtained from an IMU (Inertial Measurement Unit). The key idea is to estimate these uncertain parameters rather than treat them 
as random measurement errors.

\section{Problem Formulation}

We consider the problem of tracking a submersible using acoustic messages that are transmitted at different times by multiple reference beacons over a period $T$. The beacons deduce their location and time from GPS. However, the submersible is not time-synchronized with the beacons. Our goal is to estimate the Maximum Likelihood (ML) position and clock offset of the submersible over a time epoch $T$ given the send and receive timestamps of acoustic messages transmitted by the beacons and IMU measurements obtained during that time. Next, we present a mathematical formulation of our tracking problem.

Each acoustic message transmitted by a beacon includes the position of the beacon as obtained from GPS and a time stamp indicating the time it was sent according to global time. The receive time of the message is recorded according to the local clock of the submersible. However, the receive time of the acoustic message (in local time) cannot be determined exactly due to noise, surface reflections and multipath. We definite the cumulative effect of these random errors as the error in the detecting the first arrival of the signal and denote it as $\varepsilon_{k}$. The statistics of this error is known from previous experimental characterizations performed in prior work [4][5]. We model the statistics as a zero mean Gaussian distribution with standard deviation $\sigma_{R}$.

The difference in the send and receive times of the acoustic message relates the unknown position of the submersible and its unknown clock offset, as given by equation 1 .

$$
\left\|P_{i}\left(t_{k}\right)-P\left(t_{k}\right)\right\|_{2} / c=d_{i}\left(t_{k}\right) / c=T_{k, i}-t_{k}+\Delta\left(t_{k}\right)+\varepsilon_{k}
$$

where $c$ is the speed of sound in $m / s, P_{i}\left(t_{k}\right)$ and $P\left(t_{k}\right)$ denote the positions of beacon $i$ and the submersible at the time of message transmission, respectively.

155 The transmit time of the $k^{t h}$ message according to global time is denoted by $t_{k}$. The receive time of the message from beacon $i$ according to the submersible's 
local time is denoted by $T_{k, i}$. We refer to $T_{k, i}-t_{k}$ as the measured time-of-flight of the acoustic message and denote it as $m_{i}\left(t_{k}\right) . \Delta\left(t_{k}\right)$ is the unknown clock offset of the submersible at the time of message transmission.

Acoustic messages are received from multiple beacons over a time epoch, $T$ as depicted in Figure 1(b). Contrary to the assumption made by TDoA, the submersible's clock-offset and position both vary during the time epoch $T$.

In particular, the clock-offsets at any two time instances $t_{k}$ and $t_{k+1}$ are related to each other via the clock drift, denoted as $\eta$, as per the following equation:

$$
\Delta\left(t_{k+1}\right)=\Delta\left(t_{k}\right)+\eta \cdot\left(t_{k+1}-t_{k}\right)
$$

In addition, the submersible's motion may be significant during the signaling period. Therefore, we take this motion into account by using measurements of the submersible's velocity that are obtained from an on-board IMU. These measurements relate the position of the submersible at consecutive time steps as follows:

$$
\tilde{v}_{k}=\left(P\left(t_{k+1}\right)-P\left(t_{k}\right)\right) /\left(t_{k+1}-t_{k}\right)+\varepsilon_{v, k}
$$

where $\varepsilon_{v, k}$ is the error in velocity measurements.

The problem that we address is to track the unknown position and clock offset of the submersible using measurements obtained in intervals of duration T. As depicted in Figure 1(b), this interval is chosen as the minimum time in which acoustic messages are received from at least four unique beacons.

Note that TDoA based localization assumes that both the position of the submersible and the clock offset remain constant during this time. This amounts to making two assumptions in our more generalized description of the problem. The first assumption is that the cumulative effect of the clock drift, $\eta \cdot\left(t_{k+1}-t_{k}\right)$ in equation 2, is negligible in the signaling period $T$. The second assumption is that the displacement of the submersible, given by $v_{k} \cdot\left(t_{k+1}-t_{k}\right)$, in equation 3 , is negligible in the signaling period $T$. From equation 1 , we observe that if this were true, the number of unknowns does not increase with each new time-of-flight 
measurement. The resulting problem is then solvable as long as measurements this approach in Section 7.

On the other hand our proposed solution only assumes that the effect of the clock drift is negligible in the signaling period. However, it takes the motion of the submersible into account. It is important to note that the uncertainty due to clock drift and that due to the vehicle displacement do not act in the same way on the ranging uncertainty. In particular the clock offset is common (i.e., the same) to the range estimates with all the beacons, whereas the position uncertainty due to motion is different and independent for each beacon.

In the next subsection, we will motivate the assumptions made by our proposed scheme by identifying scenarios where the uncertainty due to motion and/or the clock drift become significant. We consider uncertainties greater than a meter to be significant because errors due to noise, surface reflections and refraction and even hardware are expected to be of that order [4] [5]. For this analysis we denote the lag between a pair of signals as $\delta t(\mathrm{~s})$, the submersible's

\subsection{Effect of motion}

TDoA assumes that the difference in signal arrivals correspond to difference in range estimates with beacons at a particular time instance. As a result, when the submersible is moving, the error in the $2 \mathrm{D}$ position estimate is determined by the displacement of the submersible in the horizontal plane during the signaling interval. This is because the depth of the submersible is known from pressure sensors and can be compensated for. Therefore, the error in positioning is given by $v \cdot \delta t$, where $v$ is the speed of the submersible in $2 \mathrm{D}$. We evaluated this error as a function of the signaling interval $\delta t$ for nominal submersible speeds of 0.4 210 $\mathrm{m} / \mathrm{s}$ to $2 \mathrm{~m} / \mathrm{s}$.

As shown in Figure 2, the error due to the submersible's motion becomes significant when the signaling interval, $\delta t$, increases to more than a few seconds. Further, this error can be as large as $120 \mathrm{~m}$ when the signaling interval is one 


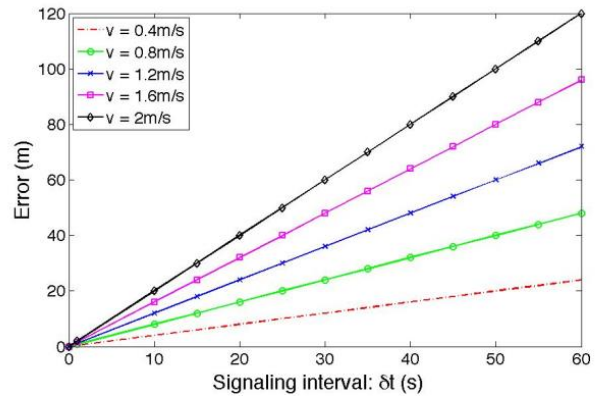

Figure 2: Error in distance due to the submersible's motion

minute long. Therefore, the effect of the submersibles motion can significantly degrade the performance of TDoA when transmissions are non-concurrent.

\subsection{Effect of clock drift}

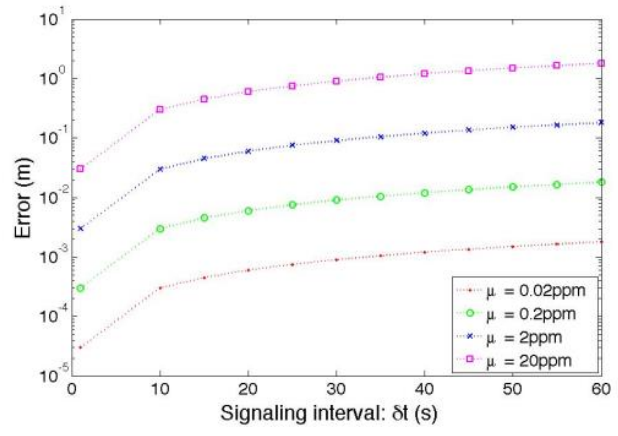

Figure 3: Error in distance due to the clock drift

Next, we consider the effect of a changing clock offset. The change in the clock offset during the signaling interval essentially translates to an error in the measured time-difference between the signal arrivals from a pair of beacons. Since the clock offset varies linearly with the drift, the change in the clock offset during the signaling interval is computed as $\eta \cdot \delta t$. When multiplied by the speed of sound, this translates to an error in (the difference in) range estimates with the beacons, given by $c \cdot \eta \cdot \delta t$. This error is shown as a function of the signaling interval in Figure 3 for clock drifts in the range of $0.02 \mathrm{ppm}$ to $20 \mathrm{ppm}$. As the 

than 20 ppm.

Based on the above results we propose that submersibles which only have receive capability incorporate low-drift clocks, especially if they are to operate in scenarios where beacon transmissions are non-concurrent. Consequently, the clock drift does not have to be estimated over short time epochs, which makes the estimation problem more tractable. Low-drift clocks have been incorporated in a number of practical underwater platforms. For example, Eustice et al have reported integrating a temperature clock into the micro-modem, with a skew of only $0.02 \mathrm{ppm}[6]$. Similar levels of accuracy have also been achieved through a novel and inexpensive approach using two crystals [7].

While the effect of clock drift is negligible when an accurate clock is used, the effect of the motion can still be significant. Although the error introduced due to motion cannot be eliminated entirely, we propose to compensate for motion as much as possible by adding of an IMU and further tracking the submersibles have an on-board IMU for tracking their location underwater. However, TDoA does not use information from an IMU, per se.

Based on the above reasoning, we consider the use of both an on-board IMU as well as a low-drift clock crystal to jointly track and synchronize a submersible. 245 Next, we present our proposed Time of Arrival based Tracked Synchronization (ToA-TS) technique. Our goal is to extend GPS like localization for systems where beacon transmissions do not occur concurrently and submersibles are not capable of two-way communication. Therefore, we propose to estimate the Maximum Likelihood (ML) positions of the submersible and a single unknown clock offset during the interval $T$. This process is periodically repeated to track and synchronize the submersible over longer durations. In the next section we describe our proposed solution. 


\section{Solution Strategy}

Our goal is to estimate the Maximum Likelihood (ML) position and clock

255 acoustic messages transmitted by the beacons and IMU measurements obtained during that time. We assume that the depth is known from pressure sensors, therefore, we estimate the position of the submersible in two dimensions.

The timestamps obtained from acoustic messages impose a non-linear and non-Gaussian constraint on the pdf of the position and clock offset of the submersible at each point in time, as given in equation 1. To incorporate such type of constraints, we appropriately define the tracking problem in the Bayesian estimation framework of factor-graphs. Factor graphs offer a way to represent any global function (in this case the joint probability distribution of the unknown position and clock offset) in terms of simpler local functions that depend only on a subset of variables. The sum-product algorithm can operate on this graph and exploit these simple relations to estimate the pdf of individual states in the graph via iterative message passing [8] [9]. We have previously discussed this framework in the context of underwater tracking [10]. However, in our previous work we used this framework to track submersibles from inter-vehicle measurements of distance. Distance estimation and time synchronization were performed independently of tracking. Here we use the framework of factorgraphs to jointly track and time-synchronize a submersible. Next, we present the factor-graph description of our tracking problem.

\subsection{Factor-graph Description}

As a first step towards solving our joint tracking and synchronization problem, we have come up with the appropriate factor-graph description given in Figure 4. The graph gives a description of the interdependencies between the unknown positions and clock offset of the submersible over time. Mathematically, this graph describes the joint distribution of the unknowns given all measurements of time-of-flight (prior to synchronization) and measurements of the 
submersible's motion in the interval $T$. Each state $P(t)$ in the factor graph (denoted by a circle) represents the unknown position of a submersible at a time instance $t$. The unknown clock offset is denoted as $\Delta(t)$ and represented by a hexagon. The square blocks, known as function-nodes, link the statevariables. The function-nodes not only indicate which state-variables are related but also how they relate. More specifically, a function node $f$ that has links to state-variables $X$ and $Y$ assigns a weight $f(X=x, Y=y \mid m)$ to any outcome $X=x, Y=y$ given a measurement $m$. Therefore, function-nodes define constraints (or interdependencies) between state-variables. Two types of constraints are captured by the graph. Function-nodes of type $f_{1}$ define constraints on the position and clock offset at each point in time as imposed by the measured time-of-flight of acoustic messages. Function-nodes of type $f_{2}$ describe how the unknown positions vary over consecutive time steps given measurements of the submersible's velocity. The clock offset remains constant over the time epoch $T$, therefore, it is represented by a single unknown state.

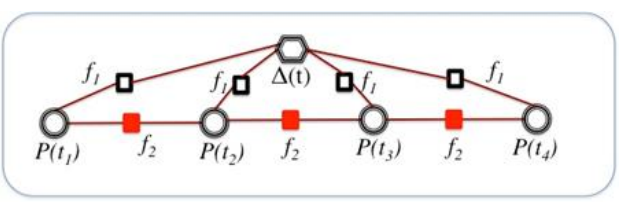

Figure 4: Factor-graph description for joint synchronization and tracking

The key advantage of the factor-graph framework is that function-nodes can take any generic form that best describes the likelihood of measurements given state-variables. This makes it possible to easily describe non-linear relations, such as the one given by equation 1. For our specific problem, the formal definitions of the function-nodes are given in equations 4 and 5 . These are derived from equations 1 and 3, respectively.

$$
\begin{aligned}
f_{1}(X(t)) & =p([P(t), \Delta(t)]) \\
& =p\left(P(t), \Delta(t) \mid m_{i}(t), P_{i}(t)\right) \\
& =\frac{1}{\sqrt{2 \pi} \sigma_{R}} e^{-\left(\left\|P_{i}(t)-P(t)\right\| / c-\left(m_{i}(t)+\Delta(t)\right)\right)^{2} / 2 \sigma_{R}^{2}}
\end{aligned}
$$




$$
\begin{aligned}
f_{2}\left(P\left(t_{k}\right), P\left(t_{k+1}\right)\right) & =p\left(P\left(t_{k}\right), P\left(t_{k+1}\right) \mid \bar{v}_{k}\right) \\
& =\frac{1}{\sqrt{2 \pi} \sigma_{v}} e^{\left\|P\left(t_{k+1}\right)-\left(P\left(t_{k}\right)+\bar{v}_{k} \cdot\left(t_{k+1}-t_{k}\right)\right)\right\|^{2} / 2 \sigma_{v}^{2}}
\end{aligned}
$$

As depicted in Figure 4, the resultant factor-graph representation of this problem is cyclic. This poses a key challenge for the tracking algorithm that which we will later describe in Section 5.2, is not guaranteed to converge for cyclic graphs.

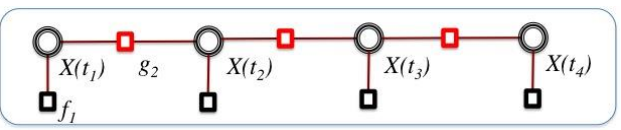

Figure 5: Acyclic factor graph obtained the stretching transformation

To address this problem we use a result by Kschischang et. al. to transform a cyclic factor-graph to an equivalent graph that is free of cycles (Section VI. $\mathrm{B}[8])$. Specifically we apply a stretching transformation to the factor-graph in Figure 4 , to obtain an equivalent acyclic graph that is shown in Figure 5. This transformation entails augmenting each of the unknown position states $P(t)$, described in $2 D$, to include the unknown clock offset, $\Delta(t)$. In effect, $\Delta(t)$ is stretched along the path to each unknown position state. The augmented state $3 D$. As a final step, we have to modify the function-nodes $f_{2}$ (in the original graph) to a new function-node, $g_{2}$ which takes as input the augmented state $X(t)$. The function node $g_{2}$ is $f_{2}$ predicated by the fact that the value of the clock offset does not change over time. $g_{2}$ is defined in equation 6 .

$$
\begin{aligned}
g_{2}\left(X\left(t_{k}\right), X\left(t_{k+1}\right)\right) & =p\left(X\left(t_{k}\right), X\left(t_{k+1}\right) \mid \bar{v}_{k}\right) \\
& =f_{2}\left(P\left(t_{k}\right), P\left(t_{k+1}\right)\right) \cdot I\left(\Delta\left(t_{k}\right), \Delta\left(t_{k+1}\right)\right)
\end{aligned}
$$


where, $I$ is a predicate function given by:

$$
I(u, v)= \begin{cases}1, & \text { if } u=v \\ 0, & \text { if } u \neq v\end{cases}
$$

The sum-product algorithm runs on the above-described factor-graph to estimate the pdf of the unknown state-variables. The algorithm in its generic form is discussed in previous tutorials [8][9]. Here we present a brief overview and examine its operation in the context of our joint tracking and synchronization problem.

\subsection{Tracking Algorithm}

Once the factor graph is defined, an iterative message passing algorithm, namely, the sum-product algorithm runs on it to solve the estimation problem. In each iteration of the algorithm, nodes in the factor-graph compose messages that are sent over the links of the graph. Messages are composed based on those received in a previous iteration.

There are two main types of messages exchanged during a single iteration of the algorithm. Messages from a function node $f$ to a state-variable $x$, denoted as $\mu_{x-f}($.$) and messages from a state-variable x$ to a function node $f$, denoted as $\mu_{f-x}($.$) . These are computed as per equations 7$ and 8 [8]. Messages that are sent out by a state-variable to its neighbor function-nodes are the most current estimate of the state-variables probability distribution. A message sent out by a function node to its neighbor state-variable is an estimate of the state-variables probability distribution, given the probability distribution of all other statevariables that are neighbors of the function node. A function node generates its message to a neighbor state-variable by performing a marginalization of its local likelihood function (as described by equation 7). A state-variable estimates its distribution (or outgoing messages) by intersecting the individual estimates of its distribution provided by its function-node neighbors, (as given by equation 345 7) . These messages, computed locally at each node, set up the machinery to 
carry information across the graph.

$$
\begin{gathered}
\mu_{f-x}(x)=\sum_{\sim(x)}\left(f(X) \prod_{y \in n(f) \backslash\{x\}} \mu_{y-f}(y)\right) \\
\mu_{x-f}(x)=\prod_{h \in n(x) \backslash\{f\}} \mu_{h-x}(x)
\end{gathered}
$$

Where the summary operation is defined in [8].

To better understand how information obtained from acoustic messages and IMU measurements are combined to solve our specific estimation problem, we present the operation of the sum-product algorithm on the factor-graph described in the previous section. We specifically consider messages passed over three iterations of the algorithm on the sub-graph shown in Figure 6.
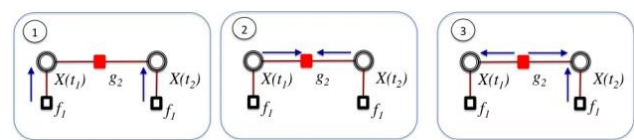

Figure 6: Operation of the sum-product algorithm on a sub-graph

Iteration1: In the first iteration, information flows from function-nodes of type $f_{1}$ to the unknown state-variables, $X\left(t_{1}\right)$ and $X\left(t_{2}\right)$ as shown in Figure 6(1). Each of these function-nodes is only linked to one state-variable. From equation 7 , the message sent from $f_{1}$ to its neighbor state-variable, $X(t)$ is $f_{1}(X(t))$, evaluated over the $3 D$ state-space of $X(t)$. This message is an estimate of the pdf of $X(t)$. Its state-space is visualized in Figure 7(a). The $x-y$ axes represent the possible positions of the submersible at time $t$, while the $\mathrm{z}$-axis represents the possible values of the clock offset. The cone shown in the figure highlights the possible position and clock offset values considered jointly, which follows directly from equation 1 . Note that the depth of the submersible is known from pressure sensors, therefore, we are effectively estimating its position in 2D. As 365 per equation 1, for a given depth of the submersible and a particular value of the clock offset, the position of the submersible is a ring in $2 D$ centered at the position of the beacon. The minimum possible radius is zero since the true time-of-flight (or distance) is always positive. As the value of the possible clock 
offset increases, so does the radius of the ring. Therefore, we obtain a cone in $3 D$ for the state-space of $X(t)$ as shown in Figure 7 .

Following equation 4 , the message passed by $f_{1}$ is a set of weights computed for each point on the cone. This $3 D$ message can be visualized as a number of pdfs in $2 D$ stacked on top of each other. Each $2 D$ pdf is defined for a particular value of the clock offset by intersecting a plane with the cone as shown in 375 Figure 7(a), and computing a set of weights over the ring thus obtained. The pdf computed over the ring by $f_{1}$ is visualized in Figure 7(b) and computed as per equation 4 for a fixed value of the clock offset. Therefore, each measurement of time-of-flight obtained from an acoustic message results in the above-described weighted-cone as the pdf of the unknown position and clock offset at that time.

380 Iteration2: In this iteration, the estimates of the pdf of $X\left(t_{1}\right)$ and $X\left(t_{2}\right)$, from the previous iteration are communicated to the function-node $g_{2}$.

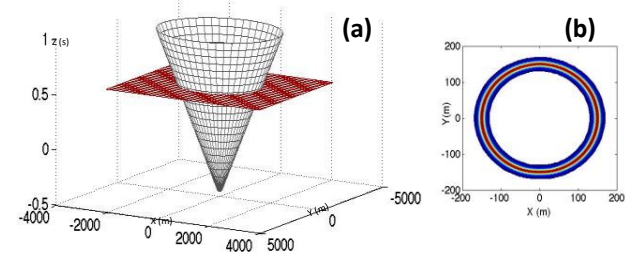

Figure 7: (a) 3D pdf of position and clock offset given the measured time of flight of an acoustic message (b) Cross-section of the pdf in 2D for a fixed value of clock offset

Iteration 3:Function-node $g_{2}$ combines these messages to estimate the pdf of each of its neighbor state-variables. The message passed from $g_{2}$ to the statevariable $X\left(t_{2}\right)$ is an estimate of the pdf of $X\left(t_{2}\right)$ given estimates of the pdfs 385 of $X\left(t_{1}\right)$. This step is essentially a $2 D$ translation and smoothing of the pdf of $X\left(t_{1}\right)$, as dictated by the velocity measurements and the statistics of error in these measurements, to obtain an estimate of the pdf of $X\left(t_{2}\right)$. The pdf of $X\left(t_{1}\right)$ is a weighted-cone (from iteration 2). Therefore, the message sent from $g_{2}$ to $X\left(t_{2}\right)$ is also a weighted-cone. A similar transformation is applied by $g_{2}$ 
compute an estimate of the pdf of $X\left(t_{1}\right)$

At the end of the third iteration, each of the state-variables $X\left(t_{1}\right)$ and $X\left(t_{2}\right)$ has two independent estimates of its pdf on its incoming links, which can be visualized as two different weighted-cones. As a final step, each state-variable intersects these pdfs to obtain a refined estimate of its pdf in $3 D$. This operation is visualized in Figure 8. The crucial point is that at the end of this step the possible values of the clock offset and position are constrained only to the regions where the cones intersect, consequently reducing the uncertainty in these estimates.

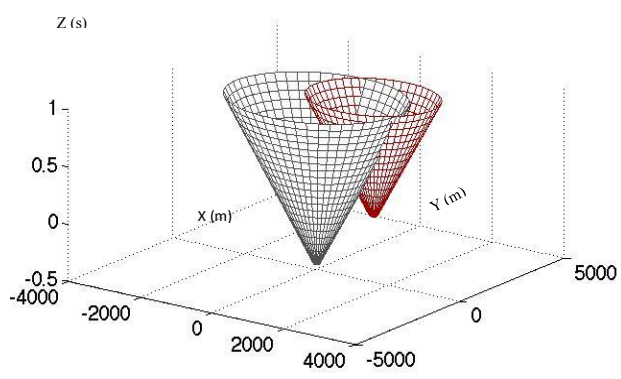

Figure 8: Reduction in the uncertainty of position and clock offset by intersecting two independently computed cones in $3 \mathrm{D}$

\section{Improvements to the algorithm run-time}

One of the key factors that determines the run time of the proposed factorgraph solution is specifying the $3 D$ space over which the pdf of the unknown position and clock offset is estimated. This can be best visualized by considering the pdf of the unknown position and clock offset in $3 D$, given a single measurement of time-of-flight as depicted in Figure 7(a). As the figure shows the pdf is specified by a cone that extends indefinitely in the positive $Z$-dimension. Estimating the pdf over this space poses a significant computational challenge.

To improve the run-time of the algorithm we would like to provide our factor graph algorithm with an initial estimate of the clock offset and the position of the vehicle for each of the states in the graph. We derive these initial estimates 

(described in Section 3). In this section we present the derivation of the least squares solution.

To obtain the least squares estimate we begin with equation 1, in Section 4. For a signal transmitted by the beacon at time $t_{k}$, this equation relates the beacon and the measured time of flight (prior to time synchronization). Squaring both sides in equation 1 results in equation 9.

$$
\left\|P_{i}\left(t_{k}\right)-P\left(t_{k}\right)\right\|^{2} / c^{2}=\left(m_{i}\left(t_{k}\right)+\Delta\left(t_{k}\right)\right)^{2}+\varepsilon_{k}^{\prime}
$$

A similar equation is obtained due to a transmission at a later time $t_{k+l}$

$$
\left\|P_{j}\left(t_{k+l}\right)-P\left(t_{k+l}\right)\right\|^{2} / c^{2}=\left(m_{i}\left(t_{k+l}\right)+\Delta\left(t_{k+l}\right)\right)^{2}+\varepsilon_{k+l}^{\prime}
$$

Subtracting equation 10 from 9, we obtain

$$
\begin{array}{r}
{\left[P\left(t_{k+l}\right)-P\left(t_{k}\right)+P_{i}\left(t_{k}\right)-P_{j}\left(t_{k+l}\right)\right]^{T}\left[P\left(t_{k+l}\right)+P\left(t_{k}\right)-P_{i}\left(t_{k}\right)-P_{j}\left(t_{k+l}\right)\right]=} \\
c^{2}\left(m_{j}\left(t_{k+l}\right)-m_{i}\left(t_{k}\right)\right) \cdot\left(m_{j}\left(t_{k+l}\right)+m_{i}\left(t_{k}\right)+2 \cdot \Delta\left(t_{k}\right)\right)+c^{2} \cdot\left(\varepsilon^{\prime}{ }_{k+l}-\varepsilon^{\prime}{ }_{k}\right)
\end{array}
$$

420

The displacement of the submersible during the period $\left(t_{k}, t_{k+l}\right)$ is computed from velocity measurments obtained during that time, as per equation 3 . Subsituting equation 3 in the above equation we obtain equation 12 .

$$
\begin{array}{r}
2\left[\left[\sum_{j=k}^{k+l} \tilde{v}_{j} \cdot \delta t+P_{i}\left(t_{k}\right)-P_{j}\left(t_{k+l}\right)\right]^{T}-c^{2}\left(m_{j}\left(t_{k+l}\right)-m_{i}\left(t_{k}\right)\right)\right]\left[P\left(t_{k}\right)^{T} \Delta\left(t_{k}\right)\right]^{T} \\
=c^{2} \cdot\left(\left(m_{j}\left(t_{k+l}\right)\right)^{2}-\left(m_{i}\left(t_{k}\right)\right)^{2}\right)- \\
{\left[\sum_{j=k}^{k+l} \tilde{v}_{j} \cdot \delta t+P_{i}\left(t_{k}\right)-P_{j}\left(t_{k+l}\right)\right]^{T}\left[\sum_{j=k}^{k+l} \tilde{v}_{j} \cdot \delta t-P_{i}\left(t_{k}\right)-P_{j}\left(t_{k+l}\right)\right]+\tilde{\varepsilon}}
\end{array}
$$

Following the approach in Section 5.1 we denote the augmented state $\left[P\left(t_{k}\right)^{T} \Delta\left(t_{k}\right)\right]^{T}$ by $X\left(t_{k}\right)$. Equation 12 is a linear equation in the unknown state $X\left(t_{k}\right)$ and can 


$$
A_{l}^{T} X\left(t_{k}\right)=b_{l}+\tilde{\varepsilon}
$$

$$
\text { where, } \begin{aligned}
A_{l}= & {\left[\left[\sum_{j=k}^{k+l} \tilde{v}_{j} \cdot \delta t+P_{i}\left(t_{k}\right)-P_{j}\left(t_{k+l}\right)\right]^{T}-c^{2}\left(m_{j}\left(t_{k+l}\right)-m_{i}\left(t_{k}\right)\right)\right]^{T} } \\
& b_{l}=c^{2} \cdot\left(\left(m_{j}\left(t_{k+l}\right)\right)^{2}-\left(m_{i}\left(t_{k}\right)\right)^{2}\right)- \\
& {\left[\sum_{j=k}^{k+l} \tilde{v}_{j} \cdot \delta t+P_{i}\left(t_{k}\right)-P_{j}\left(t_{k+l}\right)\right]^{T}\left[\sum_{j=k}^{k+l} \tilde{v}_{j} \cdot \delta t-P_{i}\left(t_{k}\right)-P_{j}\left(t_{k+l}\right)\right] }
\end{aligned}
$$

For $m$ consecutive transmissions at times $t_{k}, t_{k+1}, t_{k+2}, t_{k+m-1}$, we would obtain $m-1$ such equations. The least squares estimate for the unknown position and offset at time $t_{k}$ can be obtained as

$$
\hat{X}\left(t_{k}\right)=\left(A^{T} A\right)^{-1} A^{T} b
$$

430 where, $A=\left[\begin{array}{lll}A_{1} & A_{2} & \ldots . . A_{m-1}\end{array}\right]^{T}$ and $b=\left[\begin{array}{lll}b_{1} & b_{2} & \ldots . b_{m-1}\end{array}\right]^{T}$

The least squares estimate of the unknown position and clock-offset at each time $t_{k}$, computed as discussed above is used as an initial estimate to our factorgraph solution. This significantly minimizes the search space of the algorithm, thereby reducing the run time.

\section{Experimental Results}

To validate our approach, we applied our proposed technique to data obtained from sea trials conducted off the coast of San Diego. The experiments consisted of deploying a set of five surface buoys as shown in Figure 9. A vertical hydrophone array was allowed to drift from Buoy 1 towards the center of the deployment. The trajectory of the array is shown in the figure. Both the buoys and the receiver array were equipped with GPS. The buoys were time synchronized to GPS throughout the experiment. They also obtained their position information from GPS, which was used for localizing the array. The GPS unit on the receiver array was only used to establish ground truth position against which the tracking performance was compared. 


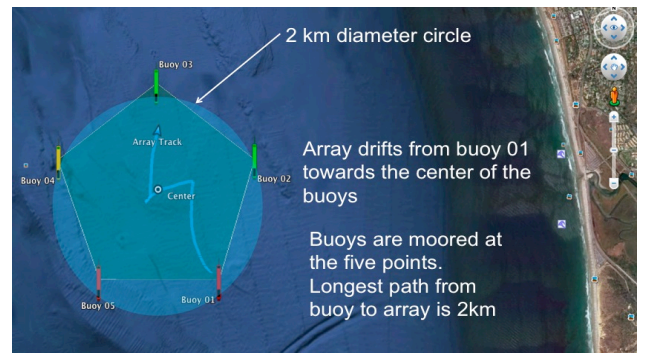

Figure 9: Experimental deployment of reference buoys and mobile vehicle

Each buoy periodically transmitted an LFM chirp in the $7-15 \mathrm{kHz}$ range. The buoy transmissions were synchronized with GPS and lagged by a second. The hydrophone array passively recorded the acoustic signals received by it. These received signals were processed post-facto. A Matched Filter approach was used to estimate the first arrival time of each signal. The location of the vehicle was estimated from the signal arrival times using three methods. In the first method, the time synchronization was done independent of the position estimation, as described in our earlier work [4]. The results of this method to the experimental data was reported in our previous work [4]. In this paper, we applied our proposed scheme to the experimental data. In this (second) method, the localization and position estimation were performed jointly using the technique proposed in this paper. Lastly, the vehicle's position was estimated using Time Difference of Arrival (TDoA). These approaches are discussed and compared in the following subsections.

\subsection{Independent Time Synchronization and Localization}

In this approach, the position of the vehicle was estimated from non-concurrent estimates of distance. Distance was estimated from measurements of time-offlight of the acoustic transmissions. To estimate time-of-flight from the recorded acoustic data we had to detect the first arrival time of each of the buoy transmissions according to the reference time of the buoys (GPS time). However, the receiver's notion of time was only available from its local clock. To translate the signal arrival times that were recorded according to local time to GPS 
time, we estimated the drift in the local clock of the vehicle. This was done by estimating the clock offset between the receiver and GPS at the beginning and end of the experiment using the signal arrival times and the known position of the vehicle from GPS (when it was on the surface and later at the end of the experiment when it resurfaced). The clock drift was then estimated by doing a linear regression on the timing offsets. The estimated drift was used to convert the signal arrival times to global (GPS) time. Time-of-flight was computed as the difference between the send and receive times of the acoustic transmissions. The speed of sound was estimated empirically from CTD profiles that were performed during the experiment. Distance was estimated as the product of the time-of-flight measurements and our estimate of the speed of sound. The error in the distance estimates was computed from the ground truth positions of the array and that of the buoys as obtained from GPS. The histogram of the error in the distance estimates is shown in Figure 10a. These errors are due to the error in our detection of the first arrival of the acoustic signals, the error in the estimate of the clock drift and the error in the estimate of the speed of sound. The estimated position of the vehicle using the above method is shown in Figure 10b.

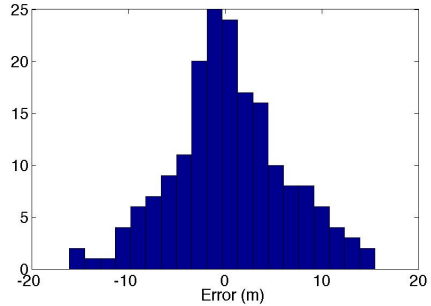

(a)

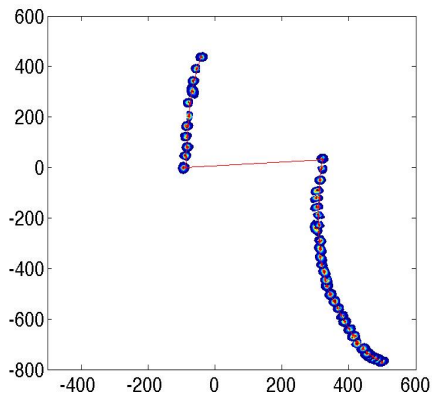

(b)

Figure 10: a) Histogram of error in distance estimates using experimental data b)Estimated trajectory, when time synchronization and position estimation were performed independently 
While such an approach provides accurate position estimates it has two main drawbacks. First, it is not amenable to a real-time solution because the clock drift can only be estimated after the vehicle resurfaces (at the end of the mission). The second drawback is that the vehicle requires to have an onboard GPS to perform time synchronization. Both these problems are addressed by our joint time synchronization and tracking technique that relies primarily on the received acoustic signals and does not require the vehicle to have a GPS. In the next section we present the results of our proposed method when applied to the experimental data.

\subsection{Joint Time Synchronization and Localization}

We estimated the position and clock offset of the vehicle jointly from nonconcurrent estimates of time-of-flight using a) the TDoA method and b) the proposed ToA-TS method. Time-of-flight was estimated as the difference between the receive time of an acoustic signal (according to the local clock of the receiver) and the transmit time of the signal according to GPS (global) time. The unknown position and clock offset were estimated from independent sets of five consecutive time-of-flight measurements. Velocity measurements were used to estimate the position at all times where distance measurements were not available. Using this approach the position of the hydrophone array was estimated every $20 \mathrm{~s}$. In the case of ToA-TS, the least squares solution proposed in section 6 was used to obtain an initial estimate which was provided as input to the factor-graph solution. This significantly reduced the (3D) space over which the pdf of the unknown position and clock offset had to be estimated by the factor-graph solution.

510

Since IMU measurements were not available in the experimental data set, velocity measurements were generated from the GPS log to emulate the presence of an IMU. However, prior to giving the velocity data to our algorithm we added randomly generated errors from a truncated Gaussian distribution with a maximum velocity error of $0.2 \mathrm{~m} / \mathrm{s}$ (the effect of varying the velocity error is 
performance of the schemes discussed so far.

7.3. Comparative Performance of Tracking and Time Synchronization Techniques

We evaluated the performance of ToA-TS, TDoA and distance based tracking using all the acoustic signals received by the hydrophone array in our experiment. This corresponded to the scenario where each beacon periodically transmitted a signal every $6 s$ with one second lag between consecutive transmissions. The cdf of the RMS error in position estimates for the three schemes is shown in Figure 11. The results show that the performance of ToA-TS and ${ }_{525}$ TDoA are similar and both these schemes outperform distance-based tracking. This is because in the distance-based solution, imprecise knowledge of the clockoffset introduces an error in the estimated distances, while ToA-TS and TDoA do not suffer from this problem because they jointly estimate the clock offset and the position of the array. Since the signals are transmitted nearly concurrently, the performance of TDoA and ToA-TS are very similar. The motion of the array during the interval that it receives measurements from all the five beacons accounts for the small difference in performance of ToA-TS and TDoA.

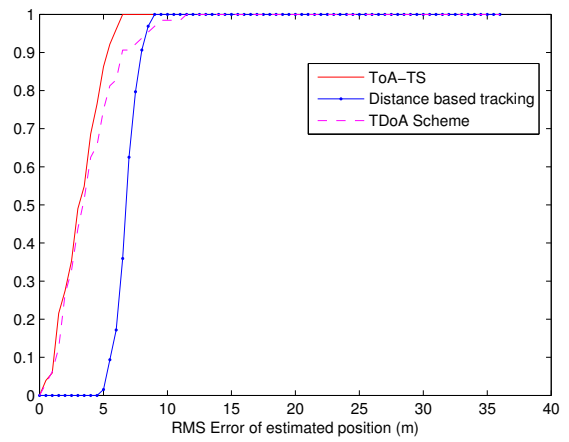

Figure 11: CDF of RMS Error in position estimates using experimental data with all measurements between signal transmissions from beacons every one second 


\subsubsection{Reducing the Beacon Signaling Frequency}

In our original experiments each beacon transmits every $6 s$ with a lag of a

every $20 \mathrm{~s}$. For these two scenarios we compared the performance of our distancebased factor graph solution (proposed in earlier work), TDoA and the proposed ToA-TS scheme.

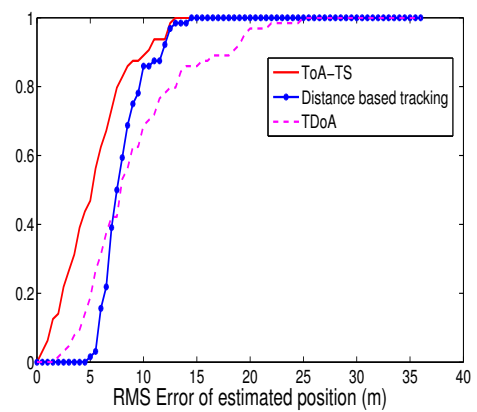

(a)

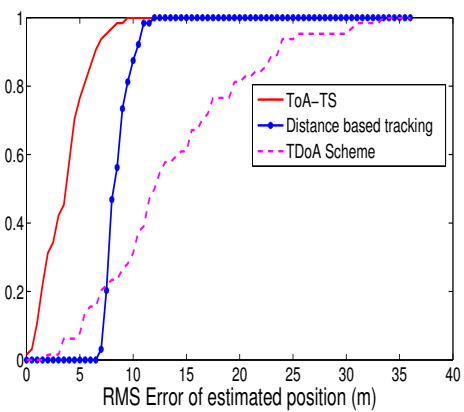

(b)

Figure 12: CDF of RMS Error in position estimates using experimental data with (a) one second lag and (b) 16 second lag between beacon transmissions

Figure 12a shows the cdf of the RMS error in the position estimates of the hydrophone array for the the different localization techniques when the signal arrivals were nearly concurrent, with a lag of only a second between consecutive transmissions. The results show that ToA-TS outperforms both TDoA and our originally proposed distance based solution. We observe that there is a consistent error gap of approximately $5 \mathrm{~m}$ between the RMS error of the position estimates obtained using ToA-TS and TDoA. This difference is attributed to the fact that TDoA assumes that the signals are transmitted concurrently in 
time. The maximum speed of the hydrophone array (as obtained from the GPS $\log$ ) was approximately $0.5 \mathrm{~m} / \mathrm{s}$, which corresponds to a displacement of $5 \mathrm{~m}$ between signal arrivals, when the transmissions are one sec apart. This explains the difference in performance between TDoA and ToA-TS. While our original distance based solution does not assume concurrent signal arrivals, the error in the estimated clock drift introduces additional error in distance estimates. This is because the estimated clock drift is used to translate the time of arrival of all signals obtained during the experiment to global time. If the error in the estimated clock drift is $e_{d r i f t}$, a timing error of $e_{d r i f t} \cdot T$ is added to the time of flight estimate of a signal that was transmitted $T(s)$ into the experiment. On the other hand, ToA-TS does not suffer from this problem because the signal arrivals do not have to be translated to global time.

Figure 12b shows the performance of the three schemes when the consecutive signal transmissions are lagged by $16 \mathrm{~s}$. For this scenario we observe a significant improvement in the performance of ToA-TS and a significant deterioration in the performance of TDoA. As before, the deterioration in the performance of TDoA is because of the unaccounted displacement of the hydrophone array between signal arrivals which increases when the interval between signal transmissions is increased from one second to $16 \mathrm{~s}$. Since ToA-TS uses velocity estimates to account for the displacement, it can actually benefit from a lagged signaling scheme. This is in line with our previous findings [4] where a lagged scheme depicted in Figure 1(b) outperformed the scheme where signal transmissions were nearly concurrent in time (as depicted by Figure 1(a)). However, in our earlier work these two signaling schemes were compared when the tracking was done based on distance measurements and time synchronization was performed independent of the tracking. The results shown in Figure 12b go on to show that a lagged signaling approach is also advantageous when the position and clock offset are jointly estimated as in ToA-TS. 
We evaluated the performance of ToA-TS when the error in velocity estimates was varied between $0.1 \mathrm{~m} / \mathrm{s}$ and $0.5 \mathrm{~m} / \mathrm{s}$. Since the maximum speed of the hydrophone is $0.5 \mathrm{~m} / \mathrm{s}$, we only consider velocity errors within that range.

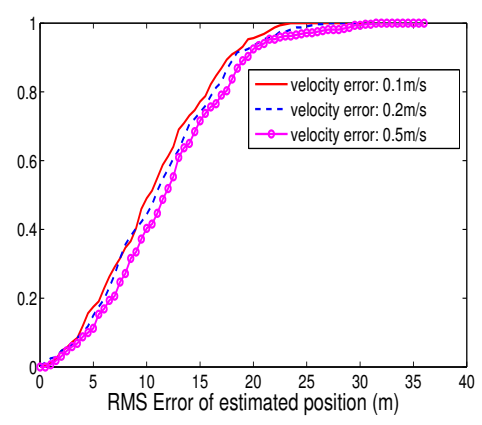

(a)

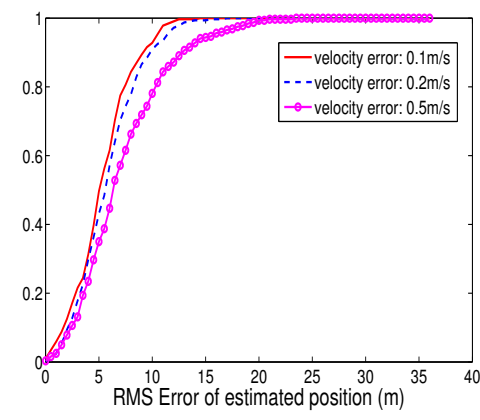

(b)

Figure 13: CDF of RMS Error in position estimates (a) 1 second lag and (b) 16 second lag in beacon transmissions when maximum velocity error is varied

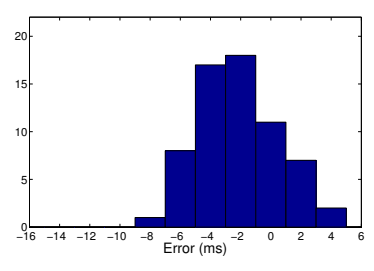

(a)

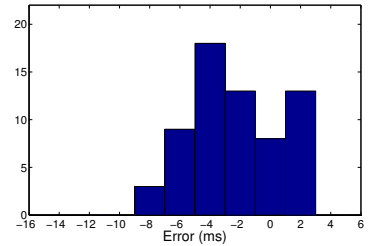

(b)

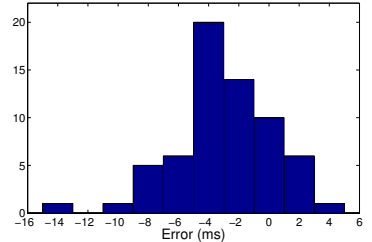

(c)

Figure 14: Histogram of error in clock offset estimates with a second lag between transmissions, max velocity error is (a) $0.1 \mathrm{~m} / \mathrm{s}$, (b) $0.2 \mathrm{~m} / \mathrm{s}$ and (c) $0.5 \mathrm{~m} / \mathrm{s}$

Figure 13a shows the RMS error in the position estimates obtained using

ToA-TS, for three different choices of velocity error: $0.1 \mathrm{~m} / \mathrm{s}, 0.2 \mathrm{~m} / \mathrm{s}$ and $0.5 \mathrm{~m} / \mathrm{s}$ and the lag between signal transmissions was one second. The histogram of the error in the estimated clock offsets for the same scenario is shown in Figure 14 (a) -(c). As expected, we observe a degradation in the accuracy of the position 


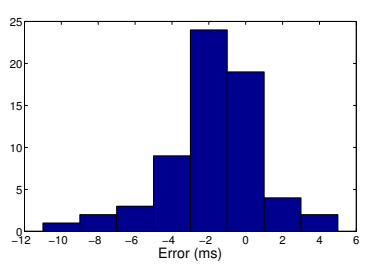

(a)

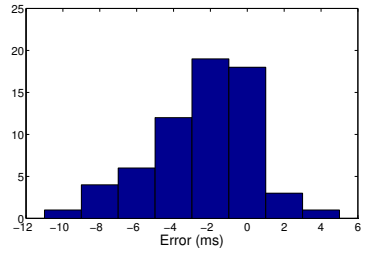

(b)

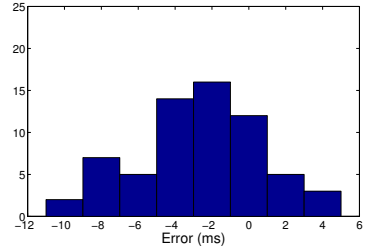

(c)

Figure 15: Histogram of error in clock offset estimates with $16 \mathrm{~s}$ lag between transmissions, $\max$ velocity error is (a) $0.1 \mathrm{~m} / \mathrm{s}$, (b) $0.2 \mathrm{~m} / \mathrm{s}$ and (c) $0.5 \mathrm{~m} / \mathrm{s}$

and clock offset estimates when the error in velocity measurements in increased.

However, this degradation is not significant.

Figure 13b shows the RMS error in the position estimates obtained using ToA-TS, when the error in velocity measurements was varied as before and the lag between signal transmissions was $16 \mathrm{~s}$. The histogram of the error in the estimated clock offsets for the same scenario is shown in Figure 15 (a) -(c). These results show a similar trend as the case where the interval between signal transmissions was a second.

In addition to the results provided in this section, we have provided extensive simulation results in the conference version of the paper [2]. In this section we validated of our proposed technique with experimental data to further support our evaluatios in simulation. The key new findings highlighted by our experimental evaluation of ToA-TS is that there is significant benefit to using ToA-TS when the rate at which beacons transmit is constrained due to energy considerations. Further, the best signaling scheme in such a scenario would be to evenly lag consecutive transmissions over the signaling period of each beacon.

\section{Related Work}

TDoA is one of the most widely used techniques to localize a submersible from one-way acoustic transmissions. Under ideal conditions, where transmissions from beacons occur concurrently, this technique serves as a way to both 
localize and time-synchronize the submersible from one-way acoustic transmissions. The TDoA approach has been extended to localizing submersibles in 3D where the depth of the vehicle is also unknown [11]. It has also been extended for the case where the beacons are not time-synchronized with each other [12]. However, these approaches do not consider the effect of vehicle motion for nonconcurrent beacon transmissions. The authors in [13] propose tracking vehicles from one way acoustic messages using a motion model rather than IMU measurements. A cooperative approach to tracking and time synchronization is proposed in [14].

Tracking and time-synchronization for mobile networks have been typically treated as separate problems [15][16]. A number of tracking techniques estimate 620 a vehicles position from non-concurrent distance estimates [10][17]. However, distance estimation requires round trip message exchange or prior time synchronization with the beacons. On the other hand, time-sync protocols that take vehicle motion into account, such as MU-Sync [18], D-Sync [19] and Mobi-Sync [20] require bi-directional acoustic signaling with beacons. Therefore, the signaling overhead associated with performing time-sync and tracking independently is substantial. In order to reduce the overall acoustic communication overhead compared to previous time synchronization protocols, JSL proposes to address the two problems jointly using a shared acoustic signaling scheme [21]. A key difference between JSL and our proposed method is that the former still uses a number of bi-directional message exchanges between the submersible and beacons while our proposed ToA-TS method is designed to work with only one-way beacon transmissions. The key motivation in the design of JSL is accounting for a depth dependent sound speed profile to correct estimates of propagation delay that are initially obtained from a straight line propagation model.

\section{Conclusion}

In this paper we have proposed ToA-TS, an approach that synchronizes a submersible while tracking it using only a few one-way acoustic transmissions. 
This method extends GPS-like time synchronization and localization for scenarios where beacon transmissions are no longer concurrent. We show that by using two simple hardware enhancements, namely an accurate clock-crystal and an on-board IMU, submersibles that can only receive acoustic transmissions can be accurately tracked and synchronized. Further, if submersibles have acoustic communication capability, our proposed approach can be applied for virtually no communication overhead on the part of submersibles.

\section{Acknowledgments}

This work is part of the project "Networked Sensor Swarm of Underwater Drifters" supported by the NSF under award number 1035828.

\section{References}

[1] K. Vickery, Acoustic positioning systems. a practical overview of current systems, in Proceedings of the Workshop on Autonomous Underwater Vehicles(AUV'98), Cambridge, MA, USA 0 (0) (1998) 5-17. doi: 10.1109/AUV . 1998.744434.

[2] J. Yi, D. Mirza, C. Schurgers, R.Kastner, Joint time synchronization and tracking for mobile underwater systems, WUWNet'13 0 (0) (2013) 0. doi : $10.1145 / 2532378.2532404$.

[3] A.Caiti, A.Garulli, F.Livide, D.Prattichizzo, Localization of autonomous underwater vehicles by floating acoustic buoys: a set-membership approach, IEEE Journal of Oceanic Engineering 30 (1) (2005) 140-152. doi : 10.1109/ JOE. 2004.841432.

[4] D.Mirza, P.Roberts, J.Yi, C.Schurgers, R.Kastner, J.Jaffe, Energy efficient signaling strategies for tracking mobile underwater vehicles, Underwater Technology 0 (0) (2013) 1-8. doi:10.1109/UT. 2013.6519841. 
[5] D.Mirza, C.Schurgers, Energy-efficient ranging for post-facto selflocalization in mobile underwater networks, IEEE Journal on Selected Areas in Communications 26 (9) (2008) 1-6. doi:10.1109/JSAC.2008. 081209.

[6] R.M.Eustice, L.L.Whitcomb, H.Singh, M.Grund, Recent advances in synchronous-clock one-way-travel-time acoustic navigation, Oceans'06 0 (0) (2006) 1-6. doi:10.1109/0CEANS. 2006 . 306931.

[7] T.Schmid, J.Friedman, Z.Charbiwala, Y.H.Cho, M.Srivastava, Xcxo: an ultra-low cost ultra-high accuracy clock system for wireless sensor networks in harsh remote outdoor environments, ISSCC/DAC08 0 (0) (2008) 0.

[8] F. Kschischang, B. Frey, H. Loeliger, Factor graphs and the sum-product algorithm, IEEE Trans. on Information Theory 47 (2) (2001) 498-519. doi : $10.1109 / 18.910572$.

[9] H. Loeliger, An introduction to factor graphs, Signal Processing Magazine 21 (1) (2004) 28-41. doi:10.1109/MSP . 2004.1267047.

[10] D.Mirza, C.Schurgers, R.Kastner, Real-time collaborative tracking for underwater networked systems, WUWNet'12 0 (0) (2012) 0. doi:10.1145/ 2398936.2398940.

[11] C.Tian, W.Liu, J.Jin, Y.Wang, Localization and synchronization for 3d underwater acoustic sensor networks, Springer-Verlag Berlin Heidelbery 0 (0) (2007) 622-631. doi:10.1007/978-3-540-73549-6_61.

[12] X.Cheng, H.Shu, Q.Liang, D.C.Du, Silent positioning in underwater acoustic sensor networks, IEEE Trans.Vehicular Technology 57 (3) (2008) 17561766. doi:10.1109/TVT.2007.912142.

[13] P. Carroll, S. Zhou, H. Zhou, X. Xu, J. Cui, P. Willett, Underwater localization and tracking of physical systems, Journal of Electrical and Computer Engineering, Special Issue on Underwater Communications and Networks 0 (0). doi : 10.1155/2012/683919. 
[14] W. van Kleunen, N. Meratnia, P. J. M. Havinga, als-coop-loc: Cooperative combined localization and time-synchronization in underwater acoustic networks, CyPhy 20140 (0) (2014) 23-26. doi:110.1145/2593458.2593462.

[15] H.P.Tan, R.Diamant, W.K.G.Seah, A survey of techniques and challenges in underwater localization, Journal of Ocean Engineering 38 (14-15) (2011) 1663-1676. doi:10.1016/j.oceaneng.2011.07.017.

[16] F.Sivrikaya, B.Yener, Time synchronization in sensor networks: A survey, IEEE Network 18 (4) (2004) 0. doi:10.1109/MNET.2004.1316761.

[17] D.Fox, W.Burgard, H. Kruppa, S.Thrun, A probabilistic approach to collaborative multi-robot localization, Autonomous Robots 8 (3) (2000) 325344. doi:10.1023/A:1008937911390.

[18] N.Chirdchoo, W.-S. Soh, K. C.Chua, Mu-sync: A time synchronization protocol for underwater mobile network, WUWNet'08 0 (0) (2008) 35-42. doi:10.1145/1410107.1410115.

[19] F.Lu, D.Mirza, C.Schurgers, D-sync: doppler-based time synchronization for mobile underwater sensor networks, WUWNet'10 0 (0). doi:10.1145/ 1868812.1868815.

[20] J.Liu, Z.Zhou, Z.Peng, J.-H.Cui, Mobi-sync: Efficient time synchronization for mobile underwater sensor networks, in Proceedings of IEEE Globecom,December 0 (0) (2010) 1-5. doi:10.1109/GLOCOM . 2010.5683527.

[21] J.Liu, Z.Wang, M.Zuba, Z.Peng, J.H.Cui, S.Zhou, Sl: joint time synchronization and localization design with stratification compensation in mobile underwater sensor networks, SECON'12 0 (0) (2012) 317-325. doi:10.1109/SECON.2012.6275793. 
${ }^{*}$ Author Biography

Jinwang Yi received a Ph.D. degree in communication engineering from Xiamen University, Xiamen, China in 2014 and a bachelor degree (B.S.) in biomedical engineering from Xidian University, Xian, China in 2007. He was a visiting $\mathrm{PhD}$ student at the department of Computer Science and Engineering at University of California, San Diego from 2011 to 2013. His research interests include sensor networks, underwater acoustic communications and signal processing. 
Curt Schurgers received his Ph.D. from UCLA in integrated circuits and systems, and his engineering degree from the Katholieke Universiteit Leuven (Belgium). He was a lecturer at UCLA in VLSI System Design, and held research assistantships at UCLA Networked \& Embedded Sysytems Lab and the Interuniversity Microelectronics Center in Belgium. He joined UCSD in 2003 as a professor in ECE, and currently works at the UCSD Qualcomm Institute as a research engineer, project leader, area manager and development engineer. 
Jules S. Jaffe is a Research Oceanographer in the Marine Physical Lab at the Scripps Institution of Oceanography, U. C. San Diego where he has worked for 25 years. He previously worked at the Woods Hole Oceanographic Institution as an Assistant and Associate Scientist. Jules received his Ph.D. From U. C. Berkeley in Biophysics, a degree in Biomedical Information Science from the Georgia Institute of Technology and a B. A. from S. U. N. Y. Buffalo in physics. He is a Fellow of the Acoustical Society of America and was a visiting Miller Professor at U. C. Berkeley. In 2012 he was awarded a best paper at the International Ocean Optics meeting. Jules is also an Associate Editor of IEEE Ocean Engineering and started a new journal in 2012 in which he is Editor-in-Chief; Methods In Oceanography. His work concerns the creation of novel instruments for measuring the organisms and the physics of the ocean. 
Diba Mirza is currectly a Lecturer in the Department of Computer Science and Engineering at the University of California, San Diego. She received a M.S and Ph.D in Electrical Engineering from UCSD in 2010 and a B.E. in Electrical Engineering from Birla Institute of Technology and Science, Pilani, India in 2002. She was a postdoc at the Department of Computer Science and Engineering at UCSD from 2010 to 2013. Her research interests include underwater acoustic navigation and networked systems. 


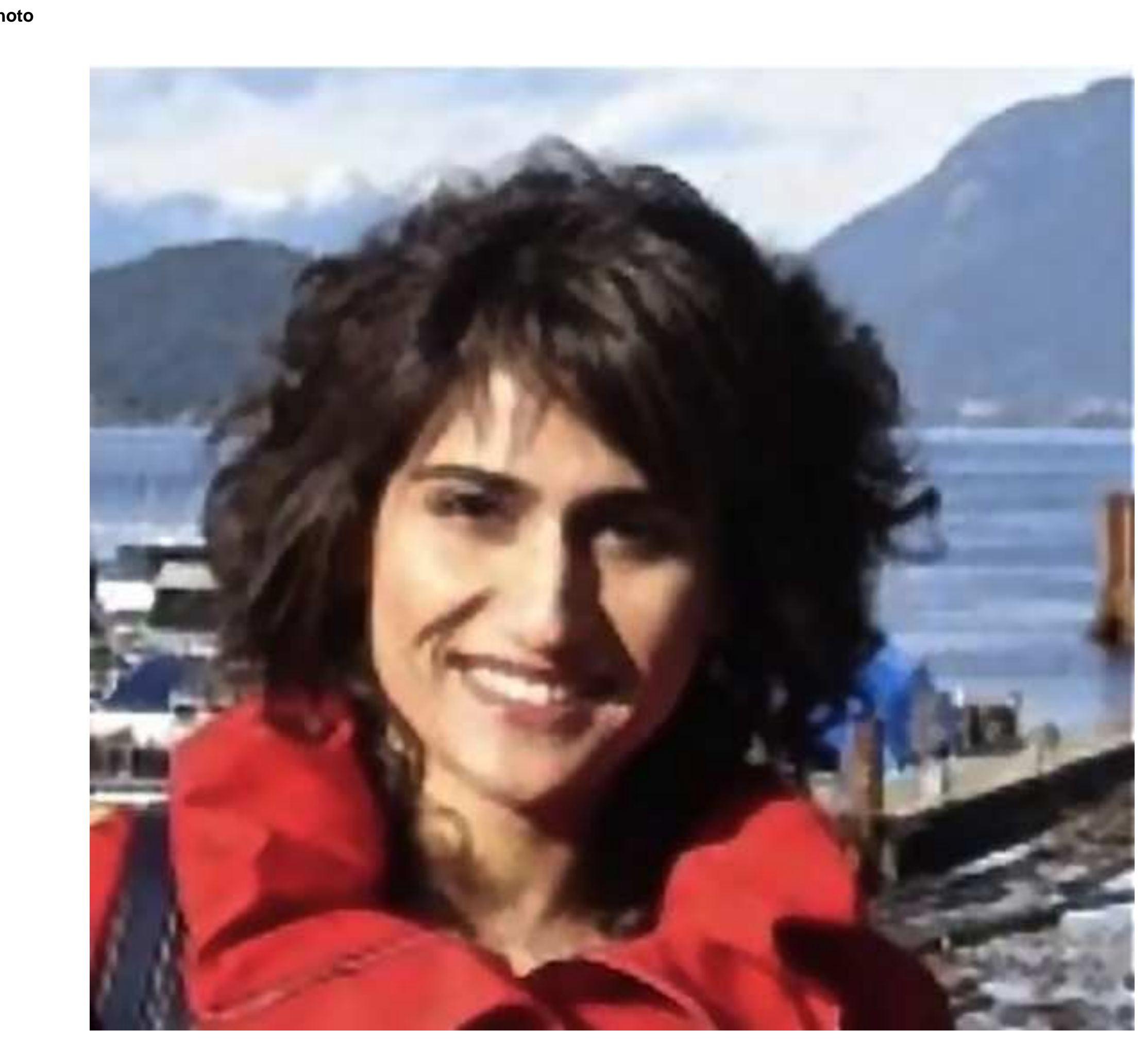

\section{Author Photo}

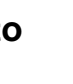




\section{${ }^{*}$ Author Photo}

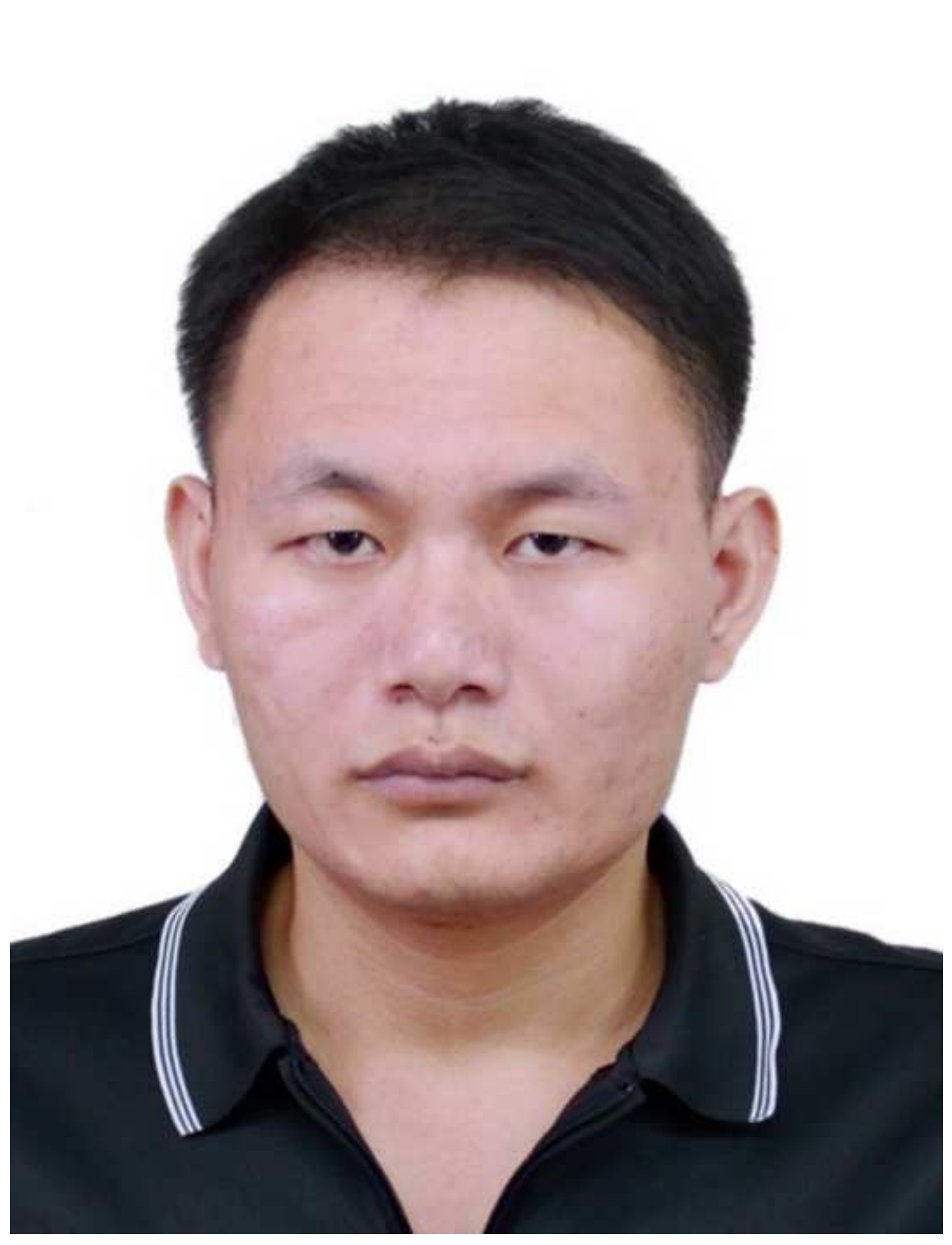

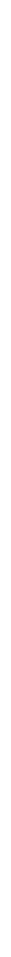
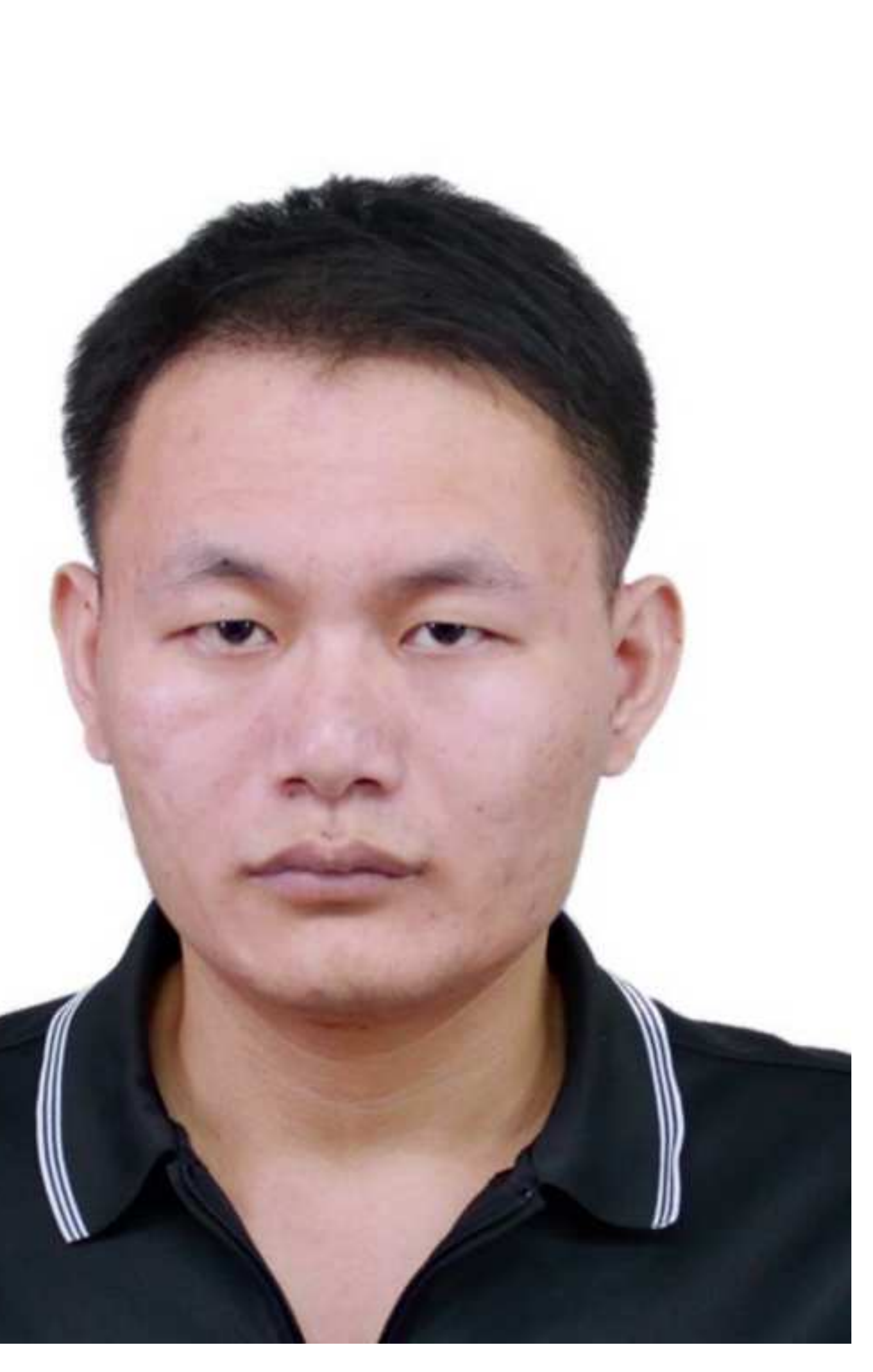


\section{*Author Photo

\section{^Author Photo}

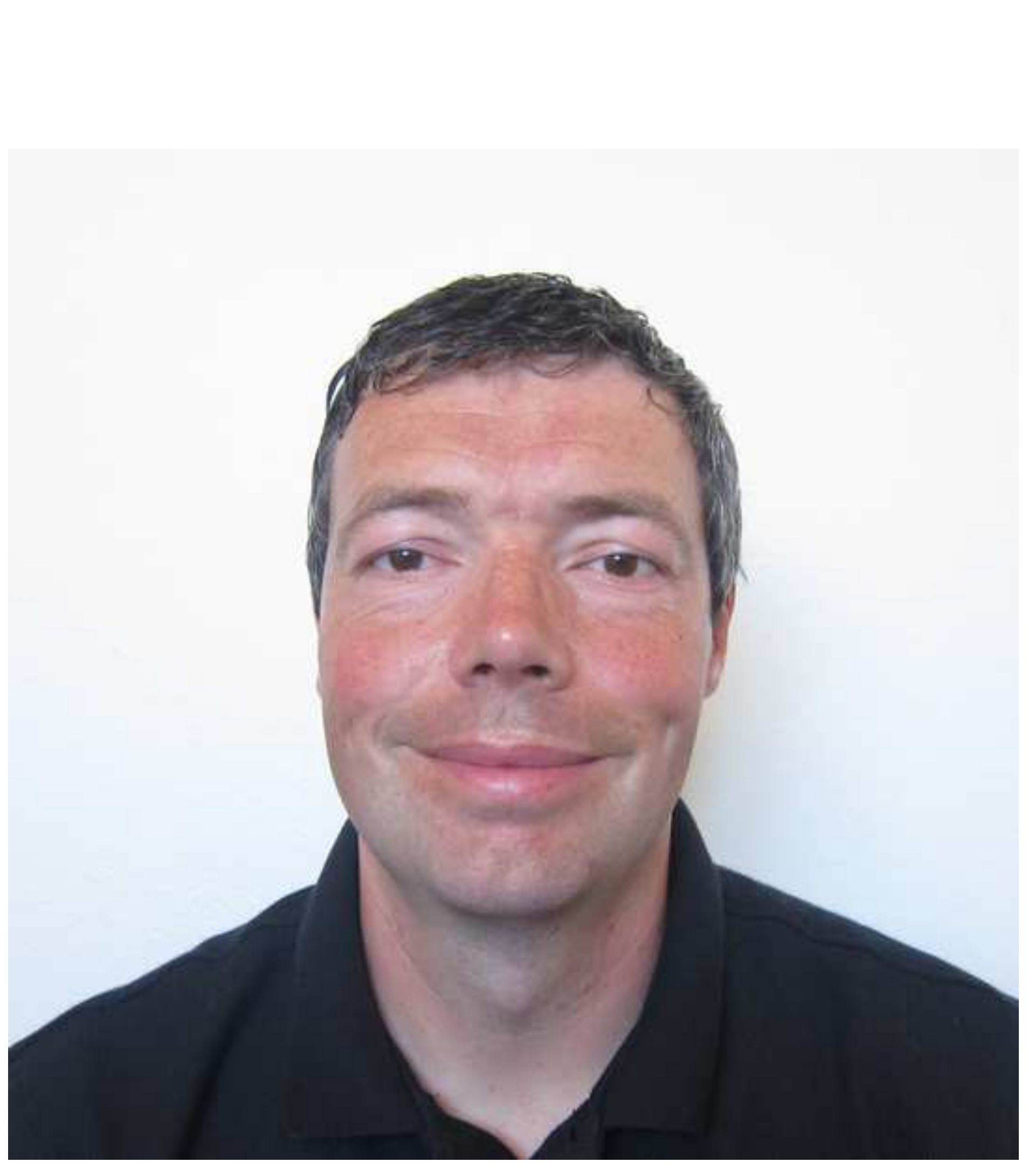

$\sqrt{2}$

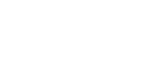

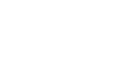

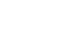

$\sqrt{2}$

$x^{2}$

(2n)
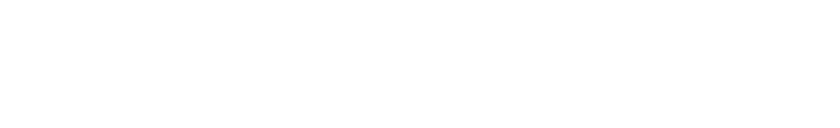


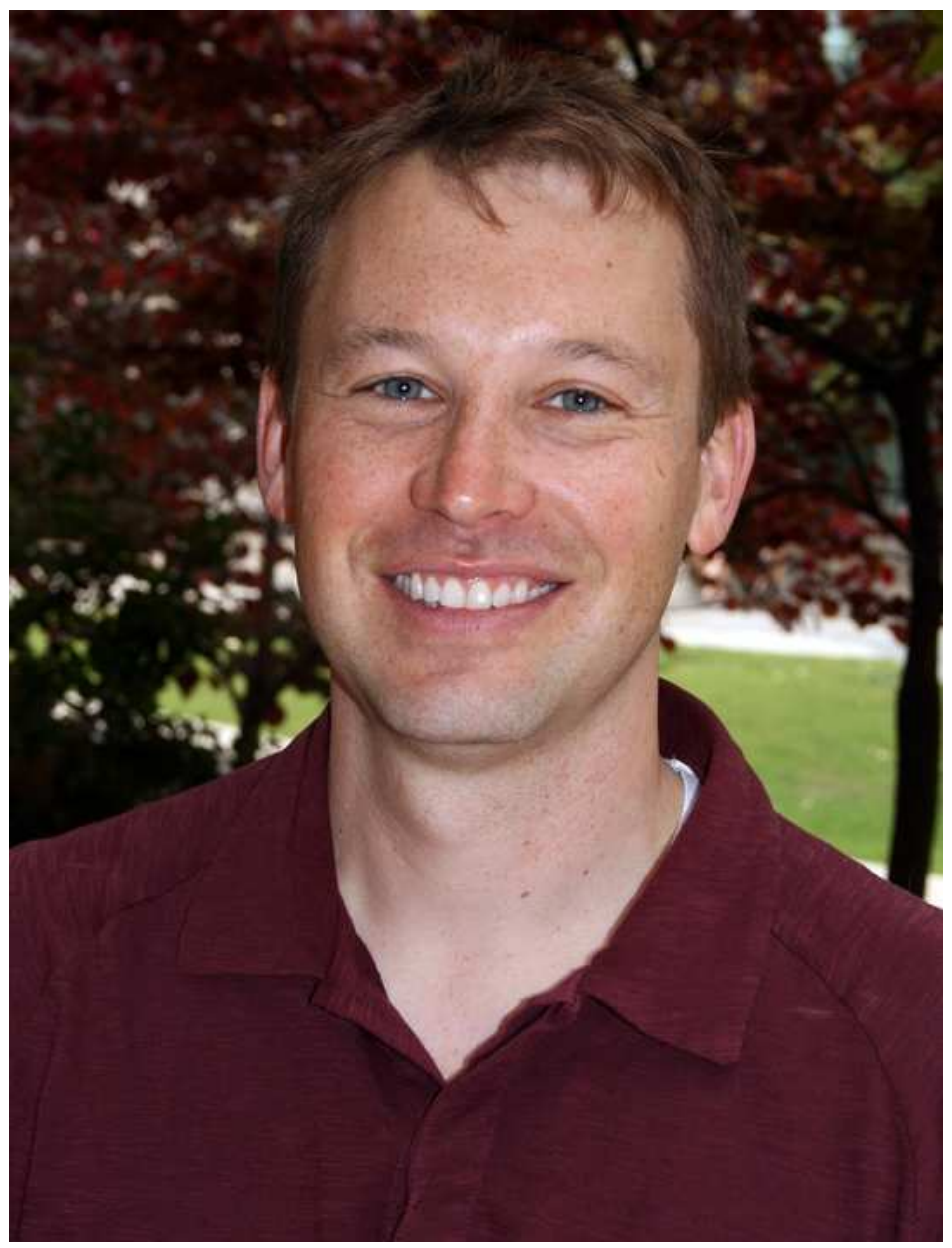




\section{*Author Photo

\section{Author Photo}
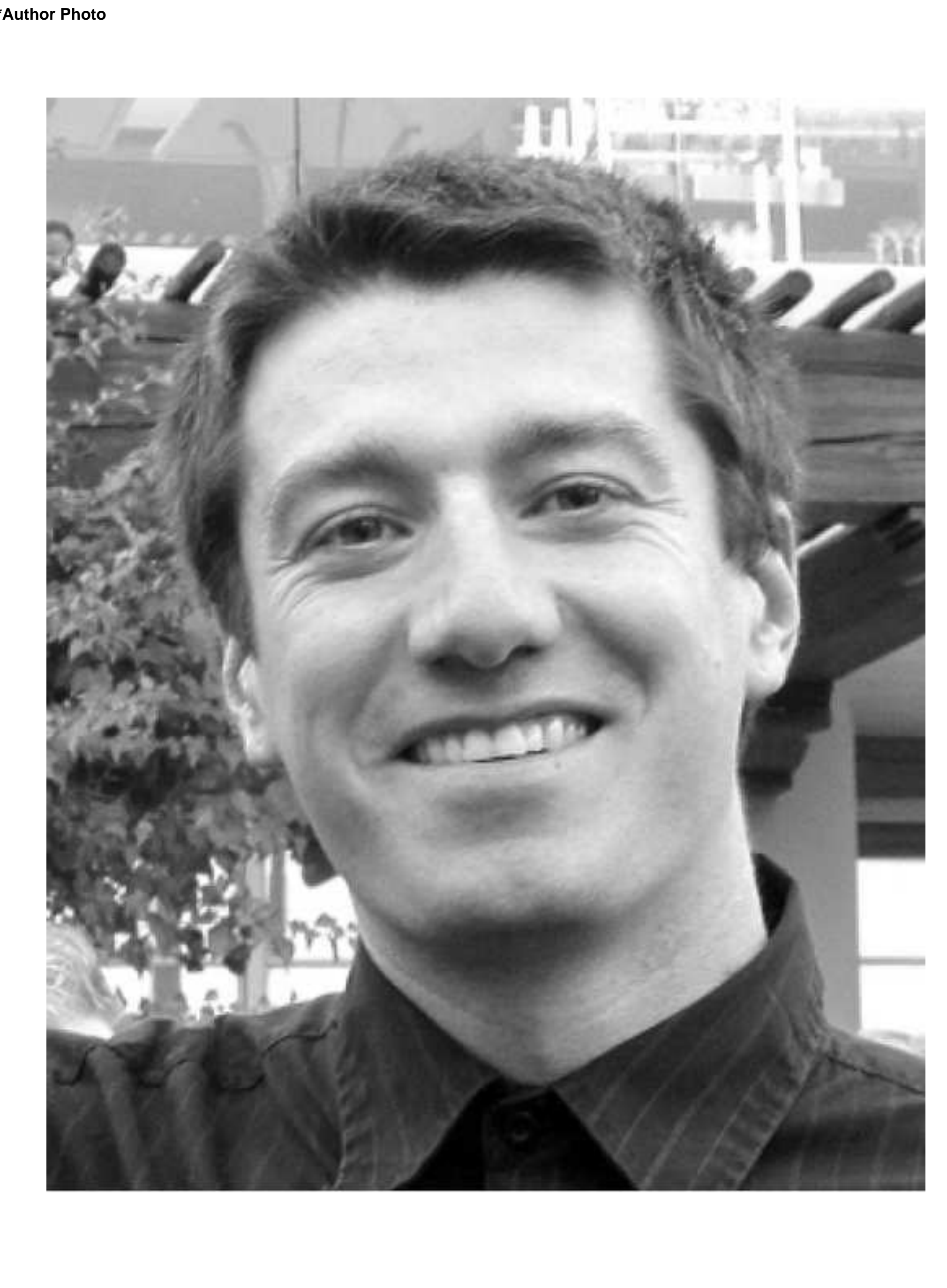

(6)
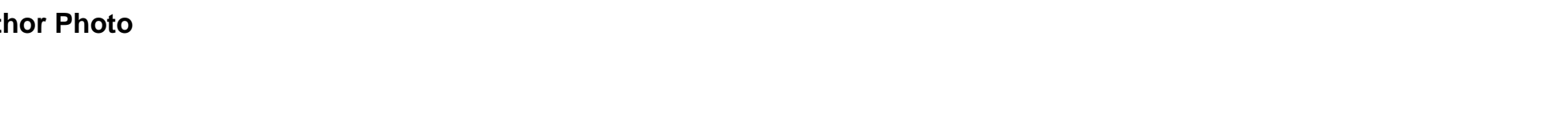

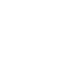

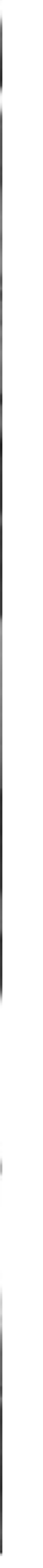

\section{Any $=5$}

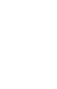


